\title{
Rare Incidental Finding on Coronary Artery CT Angiography: Left Main Coronary Artery Atresia
}

\author{
Achado Raro Incidental em Angiotomografia de Artérias Coronárias: Atresia do Tronco da Coronária Esquerda
}

Maria Eduarda Menezes de Siqueira' ${ }^{1}$ Mayra Isabel Dias ${ }^{1}$, Ana Claudia Vincezi Raudan Uski ${ }^{1}$, Luciano Figueiredo de Aguiar Filho ${ }^{1}$

${ }^{\top}$ Santa Catarina Hospital, São Paulo, SP, Brazil

\section{Introduction}

Congenital left main coronary artery atresia (CLMCAA) is a coronary anomaly in which the left coronary ostium or trunk is absent. The anterior descending (AD) and circumflex (CX) arteries are usually connected and proximally end blindly. ${ }^{1}$ Left chamber perfusion is provided by the right coronary (RC) collaterals. It is the rarest coronary anomaly, with fewer than 70 cases described in the literature. ${ }^{2}$ The clinical CLMCAA spectrum ranges from asymptomatic cases (due to an efficient collateral system) to symptoms such as angina, tachyarrhythmias, syncope, and sudden death..$^{3,4}$ Surgical revascularization is recommended in symptomatic patients; however, preventive surgical treatment is controversial in asymptomatic patients without inducible myocardial ischemia. ${ }^{1-5}$

\section{Case report}

A 51-year-old man was referred for coronary artery computed tomography $(\mathrm{CT})$ angiography to investigate atypical chest pain. The patient denied known cardiovascular risk factors. The test was performed in a 320-channel CT scanner (Aquilion One/Prism, Canon) and demonstrated CLMCAA. The $A D$ and $C X$ arteries were connected their origin at the base of a blind fundus (Figure 1A, red arrow). Blood flow entered the left coronary tree through a collateral branch of the arterial cone with independent origin in the RC sinus, which anastomosed in the middle segment of the AD artery (Figure 1B, white arrow). There was no evidence of coronary atherosclerotic disease (CAD) or stenosis. Dipyridamole myocardial resonance showed no show ischemia or fibrosis. Clinical management was chosen.

\section{Discussion}

The CLMCAA clinical diagnosis can be neglected due to the wide range of symptoms. Pediatric patients show symptoms at an early age (syncope, dyspnea, sudden death, growth failure, infarction, ventricular tachycardia) and adults begin to show symptoms (angina, dyspnea, sudden death) at an older age ${ }^{1-5}$.

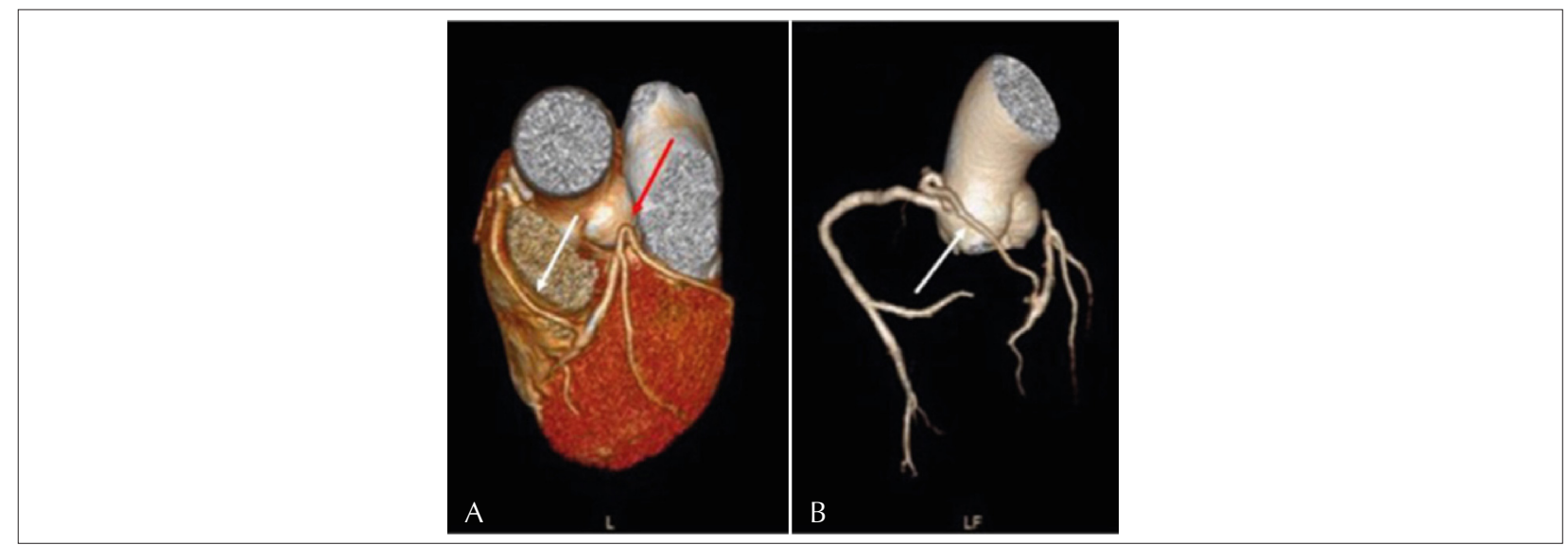

Figure 1-A. Three-dimensional reconstruction of a coronary artery CT angiogram demonstrating left main coronary artery atresia (red arrow). Figures A and B. Collateral circulation from the right coronary artery to the left circulation (white arrow).

Keywords

Anomalous LeftCoronary Artery; Tomography; Abnormality, Heart.

Mailing Address: Maria Eduarda Menezes de Siqueira •

E-mail: dadasiqueira@yahoo.com.br

Manuscript received 7/29/2021; revised 10/30/2021; accepted 11/4/2021

DOI: $10.47593 / 2675-312 X / 20213404$ eabc239 
Symptoms occur when collateral circulation from the right to the left coronary artery does not meet the cardiac metabolic needs, thus causing ischemia. Acquired coronary artery trunk (CAA) atherosclerotic occlusion has similar clinical and angiographic signs; however, in cases of true CLMCAA, the coronary arteries usually have no CAD and/or stenoses. ${ }^{1}$

CLMCAA should also be differentiated from single coronary artery (SCA). Although the RC provides the entire coronary circulation in both conditions, in CLMCAA, the left artery perfusion is retrograde and centripetal (from the periphery to the center); in SCA, the blood flow is centrifugal from the center to the periphery. Anatomically, CLMCAA is characterized by the absence of a left main trunk, which sometimes can be rudimentary, while a normal CAA is evident in SCA cases. ${ }^{5}$

CLMCAA is generally an isolated anomaly, but it may be associated with other congenital defects such as bicuspid aortic valve, supravalvular aortic stenosis, pulmonary stenosis,

\section{References}

1. Musiani A, Cernigliaro C, Sansa M, Maselli D, De Gasperis C. Left main coronary artery atresia: literature review and therapeutical considerations. Eur J Cardiothorac Surg. 1997;11(3):505-14.

2. Ryu SW, Pyo WK, Choi ES, Park CS, Yu JJ, Yun TJ, et al. Surgical treatment for left main coronary atresia with significant mitral regurgitation in a 1-year-old child. J Chest Surg. 2021;54(1):72-74.

3. Saedi S, Pouraliakbar HR, Ghaderian H, Saedi T. Congenital atresia of left main coronary artery. Egypt Heart J. 2018;70(4):451-3.

4. Singh C, Singh H, Kumar A, Banerji AK, Aggarwal N, Bharadwaj P. Congenital atresia of left main coronary artery. Indian Heart J. 2005;57(3):255-7. and ventricular septal defect. ${ }^{6}$ Noninvasive coronary CT angiography enables the diagnosis with excellent evaluation of the anatomy, collateral artery network, and associated coronary disease. As previously described, symptomatic cases or those with proven ischemia demand surgical management by myocardial revascularization. Graft patency is uncertain and requires careful follow-up. ${ }^{2}$ As it is extremely rare, CLMCAA management guidelines are lacking for it.

\section{Authors' contributions}

Manuscript writing: MEM Siqueira; critical review of the manuscript for important intellectual content: LF Aguiar; manuscript reading and review: MI Dias, ACV Raudan

\section{Conflict of interest}

The authors have declared that they have no conflict of interest.

5. Raju V, Hebbale RC, Muniswamy CS, Sivanna U, Rangaiah SKK. True congenital atresia of the left main coronary ostium: delayed presentation. Asian Cardiovasc Thorac Ann. 2018;26(1):54-6.

6. Unzué L, García E, Parra FJ, Palomo J, Friera LF, Solís J. Congenital atresia of the left main coronary artery in an adult: A rare anomaly with an unfavorable prognosis. Review of the literature. Cardiovasc Revasc Med. 2015;16(8):498-502.

7. Satran A, Dawn B, Leesar MA. Congenital ostial left main coronary artery stenosis associated with a bicuspid aortic valve in a young woman. J Invasive Cardiol. 2006;18(3):E114-6. 